should therefore be cautious about inferring too much about the nature and scope of normal human moral reasoning based solely on a small group of highly selected individuals with antisocial behaviours. In my experience, the most common and significant failures of moral reasoning happen every day in the minds of ordinary men and women.

Gwen Adshead Consultant psychiatrist, Dadd Centre, Broadmoor Hospital, Crowthorne RG45 7EG, UK. Email: gwen.adshead@wImht.nhs.uk

doi: 10.1192/bjp.bp.110.089862

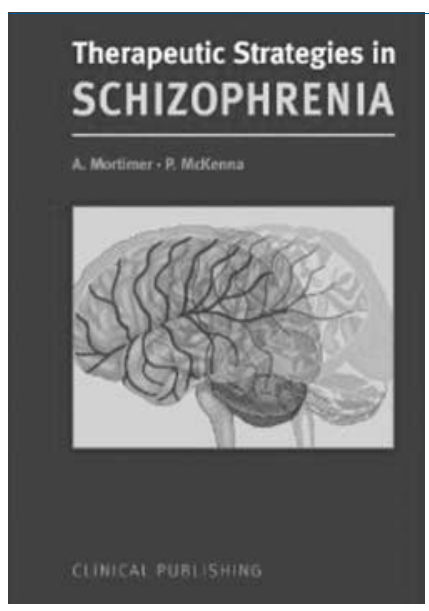

\section{Therapeutic Strategies in Schizophrenia}

Edited by Ann Mortimer \& Peter McKenna. Clinical Publishing. 2010. $£ 49.99$ (hb). 256pp. ISBN: 9781846920356

Given my work in a child and adolescent mental health servicesbased early intervention team working with adolescents and young adults with first-episode psychosis, I was interested in reviewing this book. Its collection of well-written, handily sized chapters should appeal to many working with individuals with schizophrenia. Several authors are academics but their reference to their own clinical experience suggests practice familiar within the National Health Service (NHS); a particularly pragmatic and valuable example of this is Ann Mortimer's chapter on the role and effectiveness of additional drugs in schizophrenia.

There are 14 chapters in this volume. The authors are a mix of British academics and NHS clinicians and several overseas academics. Ann Mortimer is the Head of Psychiatry at Hull University, UK and her co-editor Peter McKenna is a research psychiatrist based in Barcelona, Spain. There are several chapters on the aetiological understanding of schizophrenia, one on neurochemical theories of schizophrenia (McKenna) and two succinctly covering structural and functional brain imaging; the former, by Salgado-Pineda, Pomarol-Clotet and McKenna, addresses implications for pathophysiology, whereas the latter, from Howes and Kapur, focuses on therapeutic inferences. There is a clear emphasis on the pharmacological management of schizophrenia with individual chapters addressing medical sideeffects of antipsychotic agents (Frighi), the current status of clozapine (Kelly \& Buchanan) and disturbed behaviour and its management (Dye). A helpful brief overview of treatment strategies in early psychosis is included (Drake \& Lewis), although clinicians familiar with early intervention teams and their approaches will not find much new content here. The title wording of the chapter by Cheng and Jones I found particularly apt - 'Second generation atypical versus first-generation conventional antipsychotic drug treatment in schizophrenia: another triumph of hope over experience?' McKenna critically reviews the merit of cognitive therapy approaches in the treatment of schizophrenia and concludes, rewording text from Tarrier \& Wykes' 2004 influential paper, that cognitive-behavioural therapy use in schizophrenia should be considered 'a cautionary tale', rejecting the authors' alternative of 'cautious optimism'.

In conclusion, this volume of edited papers is an up-to-date exposition of clinically relevant themes in the contemporary management of schizophrenia, with the chapter lengths being easy to manage and the reference lists recent and thorough. This book should appeal to higher trainees anticipating working within adult psychiatry teams or destined for early intervention specialist services. Consultant psychiatrists wishing a quick up-to-date refresher of the management of schizophrenia will also find it useful.

Robert Mccabe Tier IV CAMHS (IPU \& EPSS), Royal Edinburgh Hospital Edinburgh EH10 5HF, UK. Email: robert.mccabe@nhslothian.scot.nhs.uk

doi: 10.1192/bjp.bp.110.089763

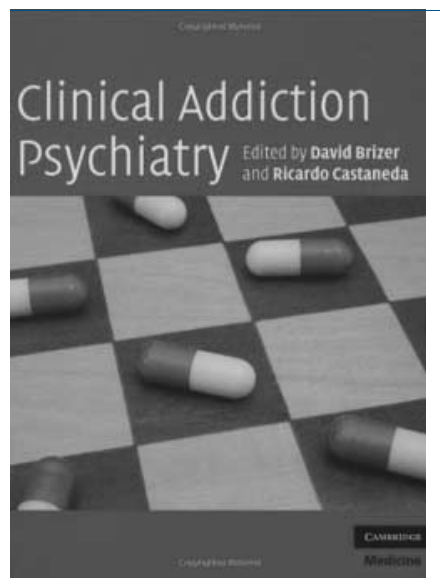

\section{Clinical Addiction Psychiatry}

Edited by David Brizer

\& Ricardo Castaneda.

Cambridge University Press. 2010.

f75.00 (hb). 268pp.

ISBN: 9780521899581

This is a multi-author book edited by two New York-based psychiatrists. All of the 30 or so contributors are from the USA. It is brief for such a textbook at around 260 pages but the list price is $\mathfrak{E 7 5}$. The back cover tells us that the book is aimed at addiction counsellors, with a hope that it will be of interest to patients, families and physicians.

Unexpectedly for a multi-author book, most of the chapters have a consistent tone and style, best described as clinical lore leavened with the occasional reference to supportive peerreviewed evidence. Only the chapters on pharmacotherapy and dialectic behaviour therapy attempt a systematic review of published evidence. There is much accumulated clinical experience described across other chapters, and the value which readers draw from this will vary depending on their own experience and whether their interests and inclinations match those of the authors. I found the chapter on cosmetic psychopharmacology, which covered caffeine, methylphenidate and steroids, interesting and novel. The section on ibogaine, a drug derived from a west African plant which has been reported to help with withdrawal and relapse prevention in opiate and cocaine dependence, was well researched and informative.

Too many of the other chapters, though, were subjective and selective for this to be recommended as a comprehensive textbook for any readership. To a UK reader, the absence of any reference to brief interventions in a chapter on substance misuse in primary care seems inexplicable. There is little on harm reduction. 
Motivational interviewing only gets a couple of passing references. The chapter on methadone gives interesting insight into the US healthcare system but, like other contributions, is more a polemic than a review. Only the dialectic behaviour therapy chapter deals in any depth with aspects of co-occurring addiction and psychiatric disorder.

For many practitioners in substance misuse and addiction psychiatry, the interface with local cultural influences is part of the fascination of the discipline. Successful international publications in addiction utilise international differences to enhance learning. This book, however, is too parochial and I could not recommend it.

Peter Rice Tayside Alcohol Problems Service, Sunnyside Royal Hospital, Montrose DD10 9JP, UK. Email: peter.rice@nhs.net

doi: 10.1192/bjp.bp.110.089789

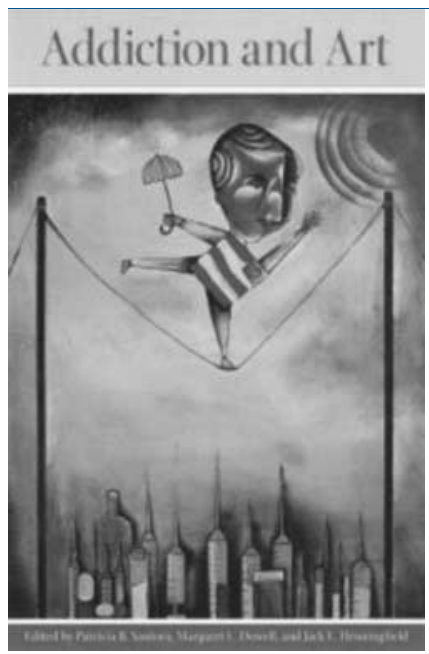

\section{Addiction and Art}

Edited by Patricia B. Santora,

Margaret L. Dowell

\& Jack E. Henningfield.

Johns Hopkins University Press.

2010.

US\$29.95 (hb). 184pp.

ISBN: 9780801894817

As the 'High Society' exhibition at the Wellcome Collection in London has shown, there is widespread interest in the influence of alcohol and drugs on our culture. If one examines Mike Jay's lavishly illustrated book of the exhibition, ${ }^{1}$ it can be seen that drug use has a multifaceted relationship with the worlds of art and literature. Much of the artwork is celebratory and indicative of the initial lure of mind-altering substances. If Jay's book presents us with the intoxication of a Saturday night, then Addiction and Art gives us the hangover of a Sunday morning, multiplied many times over by the enslavement of addiction.

The 61 stark images included in this book were selected by an expert panel from a much larger pool of work, to give insight into the desperation wrought by addictive disorders and to increase public understanding of these disorders. A further purpose was to give hope for recovery, as many of the artists are themselves recovering from addiction. Some of these images appear to be a warning to the sober self as to where a return to drug use might lead. The hopeful images contrast poignantly with those commemorating people who have died through illness, overdose or suicide. I found the painting entitled The Addiction Savior, by a Baltimore funeral director, particularly striking as a response to the epidemic of premature death wrought by tobacco, alcohol and other drugs.

Each image is complemented by explanatory text from the artist on an opposing page. These pieces then are deeply personal and give insight into a variety of individual hells. In comparison with, say, William Hogarth's Gin Lane or George Cruikshank's The Worship of Bacchus, there appears to be a lack of awareness of the social causes of the problems that are depicted. This book is very much influenced by the prevailing disease-model ideology of addictions in the USA, based on an emphasis on neurobiology at the expense of psychological and social understandings of addiction. I found myself doubting the claim put forward by the editors that science and art are reconciled here, with the art giving us the wide variety of personal experience that lies beneath the seemingly uniform surface of addictive disorders.

Ultimately, this is a worthwhile book, not only for the many striking images it presents, but also for showing how an involvement with art might for some prove therapeutic. The book does not stand alone and the project from which it is taken (www.addictionandart.org) appears to continue to thrive.

1 Jay, M. High Society: Mind-Altering Drugs in History and Culture. Thames and Hudson, 2010.

Iain Smith Gartnavel Royal Hospital, Kershaw Unit, 1055 Great Western Road, Glasgow G12 0XH, UK. Email: iain.smith@ggc.scot.nhs.uk

doi: 10.1192/bjp.bp.111.093245

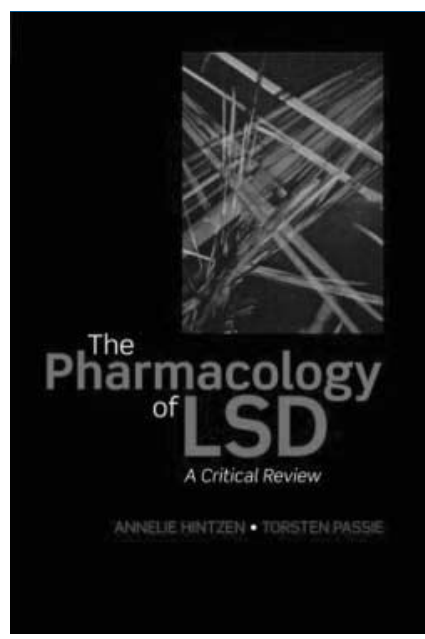

The Pharmacology of LSD: A Critical Review

By Annelie Hintzen and Torsten Passie Oxford University Press/ Beckley Foundation Press. 2010 f29.95 (pb). 240pp. ISBN: 9780199589821

Many psychiatrists today are not aware of the role lysergic acid diethylamide (LSD) played in the profession in the mid 1950s. Heralded as 'the next big thing in psychiatry', this fascinating drug looked set to transform the management of 'neurotic' patients. But in the wake of the subsequent drug revolution LSD was banned and largely consigned to museums' shelves for the past 40 years. Until now.

Psychedelic research is enjoying a worldwide renaissance with a major UK conference in April 2011 - and this book provides an impressive trawl through the database of over a 1000 pharmacological studies conducted on LSD in the past 60 years since the drug's discovery by Albert Hofmann. The book's approach to evidence for the experienced academic researcher is exhaustive, while also remaining accessible to psychiatrists and the lay enthusiast.

LSD is remarkable not least for stimulating the imagination of so many varied people. What other subject, ask the authors, spans the academic fields of biology, medicine, cultural anthropology, theology, botany, psychology and chemistry? Not to mention the drug's influence in religion, music and art.

The authors illustrate the immense potency of LSD, its pharmacodynamics and what is known of its beguiling 